\title{
Optimal Node Density for Detection in Energy-Constrained Random Networks
}

\author{
Animashree Anandkumar, Student Member, IEEE, Lang Tong, Fellow, IEEE, and Ananthram Swami, Fellow, IEEE
}

\begin{abstract}
The problem of optimal node density maximizing the Neyman-Pearson detection error exponent subject to a constraint on average (per node) energy consumption is analyzed. The spatial correlation among the sensor measurements is incorporated through a Gauss-Markov random field (GMRF) model with Euclidean nearest-neighbor dependency graph. A constant density deployment of sensors under the uniform or Poisson distribution is assumed. It is shown that the optimal node density crucially depends on the ratio between the measurement variances under the two hypotheses and displays a threshold behavior. Below the threshold value of the variance ratio, the optimal node density tends to infinity under any feasible average energy constraint. On the other hand, when the variance ratio is above the threshold, the optimal node density is the minimum value at which it is feasible to process and deliver the likelihood ratio (sufficient statistic) of the sensor measurements to the fusion center. In this regime of the variance ratio, an upper bound on the optimal node density based on a proposed 2-approximation fusion scheme and a lower bound based on the minimum spanning tree are established. Under an alternative formulation where the energy consumption per unit area is constrained, the optimal node density is shown to be strictly finite for all values of the variance ratio and bounds on this optimal node density are provided.
\end{abstract}

Index Terms-Distributed detection, error exponent, GaussMarkov random fields (GMRF), routing, sensor networks.

\section{INTRODUCTION}

W E consider the problem of distributed detection with a network of sensors that take measurements from one of the two unknown simple hypotheses, $\mathcal{H}_{0}$ and $\mathcal{H}_{1}$. Under the alternative hypothesis $\mathcal{H}_{1}$, sensors observe a Gauss-Markov random field (GMRF), and, hence, have statistically correlated samples, which in practice indicates the presence of a phenomenon of interest. Under the null hypothesis $\mathcal{H}_{0}$, on

Manuscript received October 25, 2007; revised June 5, 2008. First published July 15, 2008; current version published September 17, 2008. This work was supported in part through the collaborative participation in the Communications and Networks Consortium sponsored by the U. S. Army Research Laboratory under the Collaborative Technology Alliance Program, Cooperative Agreement DAAD19-01-2-0011 and by the Army Research Office by Grant AROW911NF-06-1-0346. The work of A. Swami was supported in part by the DARPA ITMANET program. The U. S. Government is authorized to reproduce and distribute reprints for Government purposes notwithstanding any copyright notation thereon. Parts of this work were presented at the 45th Allerton Conf. on Communication, Control and ComputingMonticello, NY,Sep.2007, and CISS '07Baltimore, MD,Mar.2007. The associate editor coordinating the review of this manuscript and approving it for publication was Dr. Danilo P. Mandic.

A. Anandkumar and L. Tong are with the School of Electrical and Computer Engineering, Cornell University, Ithaca, NY 14853 USA (e-mail: aa332@ cornell.edu; 1tong@ece.cornell.edu).

A. Swami is with the Army Research Laboratory, Adelphi, MD 20783 USA (e-mail: a.swami@ieee.org).

Color versions of one or more of the figures in this paper are available online at http://ieeexplore.ieee.org.

Digital Object Identifier 10.1109/TSP.2008.928514 the other hand, the sensors observe statistically independent measurements. Sensors deliver their measurement data to a fusion center where a test is made to determine the underlying hypothesis. We impose a constraint on the energy consumption of the fusion process, consisting of multihop routing with in-network processing. Such a fusion process can result in considerable energy savings and is in contrast to classical routing, where no processing occurs at any intermediate nodes between the source and the destination.

Classical distributed detection often makes the so-called conditional i.i.d. assumption in which observations at different sensors are statistically independent and identically distributed conditioned on each hypothesis. Such an assumption effectively decouples detection performance from energy constraints: the number of sensor measurements used for data fusion (along with optimal sensor quantization and fusion rules) determines the detection performance whereas the energy consumed in data fusion is determined by the locations of the sensors involved. Typically, one prefers to collect data from sensors near the fusion center, which implies that sensors should be densely deployed.

On the other hand, when sensor measurements are correlated, the locations of the sensors affect not only the energy consumption but also the correlation structure of the measurements. Densely deployed sensors tend to have highly correlated measurements, which may either be beneficial or detrimental to detection efficiency.

In this paper, we consider the optimization of sensor density of a multihop sensor network in the context of energy-constrained distributed detection. For large sensor networks, it is unrealistic to optimize individual sensor locations. It is then natural to consider random deployment of sensors where the sensor density becomes the key design parameter. Optimizing sensor density is not only important for sensor deployment but also gives a simple decentralized sensor transmission strategy by deciding to transmit under independent coin flips.

To characterize the detection performance, we consider the Neyman-Pearson (NP) error exponent $D$. Our objective is to find an optimal node density $\lambda_{*}$ that maximizes the detection error exponent $D_{\lambda}$, under a constraint $\bar{E}$ on the average (per node) energy consumption $\overline{\mathrm{C}}(\lambda)$, when the number of nodes goes to infinity.

$$
\lambda_{*}:=\arg \max _{\lambda>0} D_{\lambda} \quad \text { subject to } \overline{\mathrm{C}}(\lambda) \leq \bar{E} .
$$

We address the following questions: does an optimal node density exist? And if so, what is its value? Is it one of the extremes, viz., zero or infinity? This is an important question, since if the optimal node density is either zero or infinity, then we can 
simply place the nodes in as small or large an observation area as possible.

\section{RELATED WORK}

In many realistic scenarios, sensor measurements are correlated, and our framework takes this into account. In general, spatial signals are acausal in contrast to temporal signals. In the literature, the two are usually distinguished by referring to acausal signals as random fields and to causal signals as random processes. Various assumptions on spatial correlation have been made in the literature. Joint-Gaussian distributions and distancebased correlation function have been widely assumed due to their simplicity [4]-[7]. Alternatively, diffusion-based models [8] and joint-entropy based models [9] have also been employed. All these models can be incorporated under the Markov random fields (MRF) framework. Markov random fields, also known as conditional autoregressions, were introduced by Besag [10], [11]. The use of the MRF model for spatial data in sensor networks is relatively new ( e.g., [12]), although it is widely used in image processing [13] and geo-statistics [14].

Detection of a GMRF is related to the detection of a GaussMarkov random process (GMRP), which is a classical problem [15]. There is an extensive literature on the large-deviations approach to the analysis of detection of GMRP, e.g., [16] and [17]. However, GMRP is not easily applicable to random placement of nodes in planar and higher dimensional spaces. Hence, we employ the model of a GMRF for randomly placed sensors on the Euclidean plane.

We are interested in routing spatially correlated data for optimal detection and hence, there can be data fusion en route to the fusion center. Such in-network processing algorithms for computing certain aggregate functions are dealt in the surveys [18], [19]. These data-aggregation schemes, however, are applicable for optimal detection, only for conditionally independent data. An overview of routing in wireless networks can be found in [20]. The literature considering the tradeoff between detection performance and routing energy consumption is less extensive. In [21]-[23], energy-efficient detection is considered under various formulations. However, they all assume that the measurements are conditionally independent. Detection of one-dimensional GMRP is considered in [24] and [25]. A specific link cost for detection, based on the Chernoff information, is proposed in [24]. In [25], a energy-density constraint is imposed, i.e., energy per unit distance is fixed, and a finite sensor density is shown to be optimal. But these works assume a one-dimensional model, not applicable when the nodes are on a plane. In [26], nonparametric target detection is studied; binary decisions are fused locally by a majority rule before transmission to a fusion center. However, no guarantee is provided on the energy savings obtained. In [27], a decision-theoretic approach to local inference of a Markov random field with single-bit communication is considered and the network topology is predefined by a directed acyclic graph.

In [28]-[30], we consider minimum-energy fusion and energy-detection tradeoff for general Markov-random field dependencies and arbitrary node placements. We show a Steiner tree construction for the optimal scheme and a preliminary version of this work focusing on nearest-neighbor dependency appears in [3]. In contrast, an alternative formulation is considered in this paper with random point sets, which enables us to explicitly characterize scaling laws, as the number of nodes goes to infinity, for both the detection performance and the average energy consumption for data fusion, and derive optimal node density using these results. An abbreviated version of some results of this paper appears in [1]. In this paper, we provide additional results on lower and upper bounds for the optimal density under per-node energy constraint. We also consider an alternative formulation where the energy consumption per unit area is instead constrained.

\section{OVERVIEW OF RESULTS AND APPROACH}

Given a per-node energy budget for data fusion, we aim to find the sensor density that optimizes the detection performance. Assuming that all the nodes have the same measurement variance $\sigma_{j}^{2}$ under each hypothesis $\mathcal{H}_{j}$, we define the variance ratio as

$$
K:=\frac{\sigma_{1}^{2}}{\sigma_{0}^{2}}
$$

The main results demonstrate the presence of a threshold $K_{t}$ effect on the variance ratio $K$. As shown in Fig. 2, when $K$ is below a threshold $K_{t}$, the optimal density is unbounded, and thus it is optimal to concentrate sensors near the fusion center. Moreover, this result is independent of the energy constraint implying that imposing an energy constraint does not degrade detection performance. On the other hand, when $K$ is above the threshold $K_{t}$ and no energy constraint is imposed, the optimal density tends to zero [Fig. 2(a)], which in practice, implies that it is optimal to disperse sensors in the largest possible area. In this regime of $K$, imposing an energy constraint leads to a strictly finite optimal density and we provide bounds on this optimal value [Fig. 2(b)].

We give a closed-form expression for the threshold $K_{t}$ on the variance ratio $K$ which decides the behavior of the optimal density. The threshold $K_{t}$ is independent of the energy constraint. Moreover, somewhat surprisingly, it depends on the correlation structure only through the limiting correlation of two sensors as their separation distance vanishes.

We also investigate the use of an energy density constraint where the total energy consumption in a given area is constrained instead of the average energy at each node. We show that an optimal node density under this formulation exists, and is strictly finite for all values of the variance ratio $K$. This is in sharp contrast to the threshold behavior under a per-node energy constraint. We prove analytical bounds for the optimal density when the variance ratio $K<2$.

A brief comment on the approach adopted is in order. Our approach relies on the characterization of detection error exponent and energy consumption for data fusion when the sensors are randomly deployed with constant density. Note that in general, both the error exponent and the energy consumption are indeed governed by the entire correlation structure. But we prove that for a Markov random field model these quantities are only dependent on the "local" correlation behavior. The key technique is the law of large numbers (LLN) for functionals on random graphs, recently obtained by Yukich and Penrose [31]. 
In essence, the LLN states that the limit of a global functional on a random graph tends to a "local" effect around the origin under some conditions. Using the LLN, we have derived a closed-form expression for the detection error exponent in [2].

In this paper, we use the LLN to derive scaling laws for energy consumption involved in data fusion for detection. Finding the optimal fusion scheme with minimum energy consumption is intractable. We have shown that this is equivalent to a Steiner tree, and, hence, is NP-hard for any arbitrary node placement [28], [29]. Here, we analyze data fusion of a Markov random field (DFMRF), a 2-approximate fusion scheme, i.e., with energy consumption no more than twice that of the optimal scheme. This analysis is significant on its own because the DFMRF fusion scheme is implementable in practice and results in significant savings in energy consumption. In this paper, we strengthen the approximation guarantee of the DFMRF scheme for random node placement from 2 to a constant that depends on the channel path-loss exponent using the LLN. In the process, we also prove that the DFMRF fusion scheme has a constant asymptotic average energy consumption, in contrast to the classical approach of sending all the raw data to the fusion center on shortest paths, which has an almost linear scaling, as depicted in Fig. 5. Finally, we employ this scaling result as a vehicle to provide bounds on the optimal node density when the variance ratio $K$ is above threshold $K_{t}$, as seen in Fig. 2(b).

Our paper is organized as follows. The system model is explained in Section IV. The detection problem is specified in Section V and minimum energy routing in Section VI. Asymptotic analysis of routing energy and detection performance is provided in Section VII. Results on optimal density are in Section VIII and numerical analysis in Section VIII-D. The energy-density formulation is dealt in Section IX. Section X concludes the paper.

\section{SYSTEM MODEL}

An undirected graph $\mathcal{G}$ is a tuple $\mathcal{G}=(\mathcal{V}, \mathcal{E})$ where $\mathcal{V}$ is the vertex set and $\mathcal{E}=\{(i, j), i, j \in \mathcal{V}, i<j\}$ is the edge set. Let $R_{i j}$ be the random variable denoting the Euclidean edge-length of $(i, j)$. The neighborhood function of a node $i$ is the set of all other nodes having an edge with it. For a directed graph, we denote the edges by $\mathcal{E}=\{\langle i, j\rangle, i, j \in \mathcal{V}, i \neq j\}$, where the direction of the edge is from $i$ to $j$.

For the matrix $\mathbf{A}=[A(i, j)], A(i, j)$ denotes the element in the $i$ th row and $j$ th column and $|\mathbf{A}|$ its determinant. For two sets $A$ and $B$, let $A \backslash B=\{i: i \in A, i \notin B\}$.

\section{A. GMRF}

A GMRF, in addition to being a Gaussian random field, satisfies special conditional independence properties. A simple example is the first-order autoregressive process, where the conditional independence of the observations is based on causality. However, a spatial random field has a far richer set of conditional independencies, requiring a more general definition [32, p. 21].

Definition $1(G M R F)$ : Given a point set $\mathcal{V}=\{1, \ldots, n\}$, $\mathbf{Y}_{\mathcal{V}}=\left\{Y_{i}: i \in \mathcal{V}\right\}$ is a GMRF with an (undirected) dependency graph $\mathcal{G}_{d}\left(\mathcal{V}, \mathcal{E}_{d}\right)$ if $\mathbf{Y}_{\mathcal{V}}$ is a Gaussian random field, and $\forall i, j \in \mathcal{V}, Y_{i}$ and $Y_{j}$ are conditionally independent given observations at all other nodes if $i$ and $j$ are not neighbors

$$
Y_{i} \perp Y_{j} \mid \mathbf{Y}_{-i j} \Longleftrightarrow(i, j) \notin \mathcal{E}_{d}, \quad \forall i, j \in \mathcal{V}, \quad i \neq j
$$

where $\perp$ denotes conditional independence and $\mathbf{Y}_{-i j}:=\left(Y_{k}\right.$ : $k \in \mathcal{V}, k \neq i, j)$.

\section{B. Network and Energy Model}

We assume that the energy consumed by a node $i$ can be represented by the sum of a constant processing energy $C_{p}>0$ and the transmission energy ${ }^{1}$ [33]. For details, see [3]. The energy required for the transmission of a real number from $i$ to $j$ is denoted by $C_{i, j}$. We assume that

$$
C_{i, j}=C_{t}|\operatorname{dist}(i, j)|^{\nu}, \quad 2 \leq \nu \leq 4
$$

where $C_{t}$ is a constant, $\operatorname{dist}(i, j)$ is the internode distance, and $\nu$ is the channel path-loss exponent. Further, we assume that the processing energy $C_{p}$ at every node is constant and independent of the number of transmissions from the node. Hence, it can be ignored in the optimization framework for average per-node energy.

The set of transmissions within a node set $\mathcal{V}$ can be represented as a digraph $\mathcal{G}_{t}=\left(\mathcal{V}, \mathcal{E}_{t}\right)$. Note that this transmission digraph $\mathcal{G}_{t}$ is different from the dependency graph $\mathcal{G}_{d}$ of the signal field. Let $\mathrm{C}\left(\mathcal{G}_{t}\right)$ denote the total transmission energy given by

$$
\mathrm{C}\left(\mathcal{G}_{t}\right):=C_{t} \sum_{e \in \mathcal{E}_{t}} R_{e}^{\nu}
$$

where $R_{e}$ is the Euclidean edge length. We require that $\mathcal{G}_{t} \subset$ $\operatorname{UDG}(\mathcal{V})$, the unit-disk graph, defined as the set of edges between any two nodes within an unit Euclidean distance of each other. We further require $\mathcal{G}_{t}$ to deliver the log-likelihood ratio (LLR) to the fusion center to ensure optimal detection, which is explained in detail in Section V.

\section{Hypothesis-Testing PROBlem}

Let $\mathcal{V}=\{1, \ldots, n\}$ be a set of $n$ nodes on the plane and let $\mathbf{Y}_{n}$ be the random vector of observation samples $Y_{i}, i \in \mathcal{V}$

$$
\mathbf{Y}_{n}:=\left[Y_{1}, \ldots, Y_{n}\right]^{T}
$$

The hypothesis-testing problem is

$$
\mathcal{H}_{0}: \mathbf{Y}_{n} \sim \mathcal{N}\left(\mathbf{0}, \sigma_{0}^{2} \mathbf{I}\right) \text { versus } \mathcal{H}_{1}: \mathbf{Y}_{n} \sim \mathcal{N}\left(\mathbf{0}, \mathbf{\Sigma}_{\mathbf{1}, \mathcal{V}}\right)
$$

where the covariance matrix $\boldsymbol{\Sigma}_{\mathbf{1}, \mathcal{V}}$ of a GMRF depends on the configuration of nodes in $\mathcal{V}$. See Fig. 1(b) and (c).

The optimal decision-rule under the Neyman-Pearson formulation is a threshold test based on the LLR. Let $p\left[\mathbf{Y}_{n} \mid \mathcal{V} ; \mathcal{H}_{j}\right]$ be the conditional PDF of the observations given the point set $\mathcal{V}$ under hypothesis $j$, for $j=0,1$. Then, the LLR is given by

$$
\operatorname{LLR}\left(\mathbf{Y}_{n}, \mathcal{G}_{d}\right):=\log \frac{p\left[\mathbf{Y}_{n}, \mathcal{V} ; \mathcal{H}_{0}\right]}{p\left[\mathbf{Y}_{n}, \mathcal{V} ; \mathcal{H}_{1}\right]}
$$

\footnotetext{
${ }^{1}$ We ignore the energy consumed at the receiver.
} 


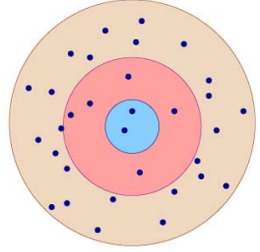

(a) $n$ nodes in area $\frac{n}{\lambda}$ Asymptotic: $n \rightarrow \infty$ Constant density $\lambda$

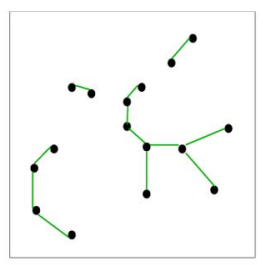

(b) $\mathcal{H}_{1}:$ GMRF, nearest-neighbor dependency graph

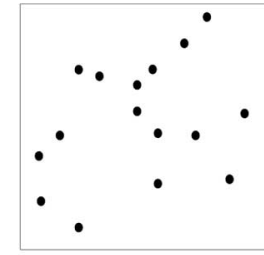

(c) $\mathcal{H}_{0}:$ Gaussian i.i.d
Fig. 1. Detection problem and random node deployment.

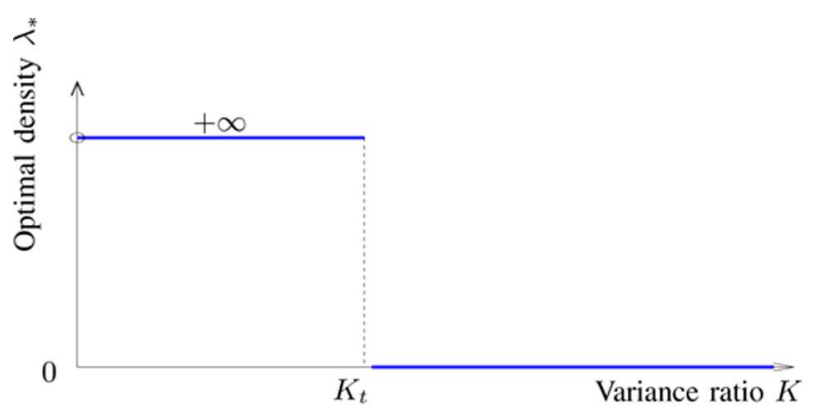

(a) No energy constraint

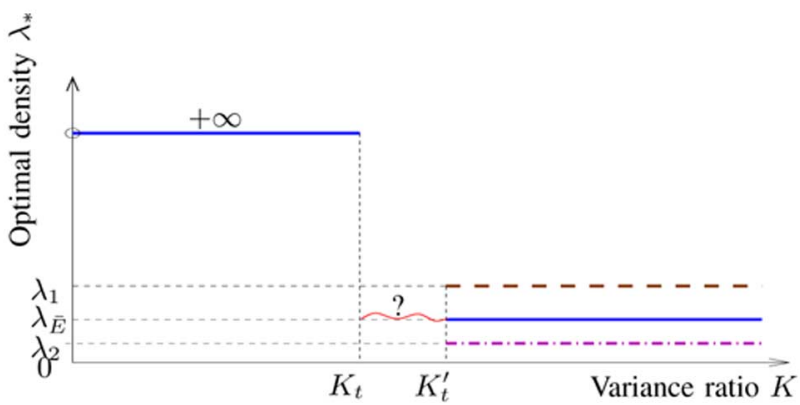

(b) Feasible energy constraint

Fig. 2. Optimal node density versus ratio of variances under $\mathcal{H}_{1}$ and $\mathcal{H}_{0}$. See Theorem 4. For a given energy constraint, $\lambda_{2} \leq \lambda_{*}=\lambda_{\bar{E}} \leq \lambda_{1}$, for $K>K_{t}^{\prime}$.

In order to conduct optimal detection at the fusion center, it suffices to deliver the LLR to the fusion center and raw data is not needed. Such data fusion schemes are outlined in Section VI.

\section{A. Covariance Matrix of GMRF}

We make an additional assumption on the structure of the covariance matrix $\boldsymbol{\Sigma}_{\mathbf{1}, \mathcal{V}}$ of the GMRF under $\mathcal{H}_{1}$ viz., that the nodes have the same measurement variance for any node configuration $\mathcal{V}$, i.e.

$$
\Sigma_{1, \mathcal{V}}(i, i):=\sigma_{1}^{2}, \quad i=1, \ldots, n
$$

We denote the ratio between the variances under the alternative and the null hypothesis at each node by

$$
K:=\frac{\sigma_{1}^{2}}{\sigma_{0}^{2}}
$$

We also assume that under $\mathcal{H}_{1}$, the amount of correlation between the neighbors ${ }^{2} i, j$ of the dependency graph is specified by an arbitrary function $g$, which has the Euclidean edge length $R_{i j}$ as its argument. From (9), we have

$$
g\left(R_{i j}\right):=\frac{\Sigma_{1, \mathcal{V}}(i, j)}{\sigma_{1}^{2}}, \quad \forall(i, j) \in \mathcal{E} .
$$

The correlation function $g$ is required to satisfy some regularity conditions. We assume that

$$
g(0):=M<1, \quad g(\infty)=0
$$

and that $g$ is a monotonically decreasing convex function of the edge length. This is a reasonable assumption, since the amount of correlation usually decays as nodes become farther apart. Moreover, partial correlation or $g(0)=M<1$ arises due to signal imperfections. Note that this has the same effect on correlation as imposing an exclusion region on how near two nodes can be placed. Some examples of the correlation functions, satisfying our assumptions, are

$$
g(R)=M \mathrm{e}^{-a R}, \quad g(R)=\frac{M}{1+R^{a}}, \quad 0 \leq a, M<1 .
$$

Note that we do not assume the presence of additive Gaussian measurement noise in the signal model, as considered in the literature before. This is because with the additive noise, the resulting GMRF no longer has a sparse dependency graph. As an alternative formulation, we model imperfect measurements by assuming partially correlated nodes.

\section{B. Nearest-Neighbor Graph}

Typically, spatial interactions are based on proximity, where the choice of edges is determined by the local point configuration according to some specified rule [34]. With a regular lattice structure (e.g., in image processing, Ising model), a fixed set of neighbors can be specified in a straightforward manner [35]. However, the situation is more complicated for arbitrarily placed nodes. In this paper, we assume the dependency graph $\mathcal{G}_{d}$ to be the nearest-neighbor graph (NNG), which is the simplest proximity graph. The nearest-neighbor function of a node $i \in \mathcal{V}$, is defined as

$$
\operatorname{nn}(i):=\arg \min _{j \in \mathcal{V}, j \neq i} \operatorname{dist}(i, j)
$$

where $\operatorname{dist}(\cdot, \cdot)$ is the Euclidean distance. The nearest-neighbor (undirected) graph $\mathcal{G}_{d}\left(\mathcal{V}, \mathcal{E}_{d}\right)$ is formally given by

$$
(i, j) \in \mathcal{E}_{d} \Longleftrightarrow i=\mathrm{nn}(j) \text { or } j=\mathrm{nn}(i) .
$$

\section{Expression for $L L R$}

We incorporate (9)-(10) on the covariance matrix in the theorem below to obtain the LLR in (8) for detection.

${ }^{2}$ Given the correlation coefficients between the neighbors of the dependency graph, the other coefficients of the correlation matrix can be inferred for acyclic dependency graphs. 
Theorem 1 (LLR): Under (9)-(10), the log-likelihood ratio in (8) for the hypothesis-testing problem in (7), given point set $\mathcal{V}=\{1, \ldots, n\}$, is shown in (14) at the bottom of the page, where $R_{i j}$ is the Euclidean edge length of $(i, j) \in \mathcal{E}$.

Theorem 1 gives a closed-form expression for the log-likelihood ratio, in terms of the edges of the nearest-neighbor dependency graph of the GMRF. Note in (14), the cross terms are only between the neighbors of the dependency graph, which is exploited to yield explicit data-fusion scheme [3], summarized in Section VI. Note that (14) contains two kinds of terms. The edge potentials, denoted by $\phi_{i, j}\left(Y_{i}, Y_{j}\right)$, that depend on a pair of observations, are given by

$$
\begin{array}{r}
\phi_{i, j}\left(Y_{i}, Y_{j}\right):=\frac{1}{2} \log \left[1-g^{2}\left(R_{i j}\right)\right]-\frac{g\left(R_{i j}\right)}{1-g^{2}\left(R_{i j}\right)} \frac{Y_{i} Y_{j}}{\sigma_{1}^{2}} \\
+\frac{g^{2}\left(R_{i j}\right)}{1-g^{2}\left(R_{i j}\right)} \frac{Y_{i}^{2}+Y_{j}^{2}}{2 \sigma_{1}^{2}}
\end{array}
$$

and the node potentials $\phi_{i}\left(Y_{i}\right)$, depending on a single observation, are given by

$$
\phi_{i}\left(Y_{i}\right):=\log \frac{\sigma_{1}}{\sigma_{0}}+\frac{1}{2}\left(\frac{1}{\sigma_{1}^{2}}-\frac{1}{\sigma_{0}^{2}}\right) Y_{i}^{2} .
$$

Hence, the LLR can be written in a compact form

$$
\operatorname{LLR}\left(\mathbf{Y}_{n} ; \mathcal{G}_{d}\right)=\sum_{i \in \mathcal{V}} \phi_{i}\left(Y_{i}\right)+\sum_{(i, j) \in \mathcal{E}_{d}} \phi_{i, j}\left(Y_{i}, Y_{j}\right)
$$

\section{Minimum Energy Routing}

The aim of optimal minimum energy data fusion for detection is to ensure the delivery of the LLR, given by (17), to the designated fusion center while minimizing the total energy consumption. First consider the special case when the measurements are independent conditioned under either hypothesis. In this case, the LLR in (17) is just the sum of the node potentials and all the edge potentials are zero. The minimum-energy routing in this case is given by the directed minimum spanning tree (DMST), with the directions toward the fusion center. See Fig. 3(a). The sum function can be calculated hierarchically along the DMST, starting at the leaves and ending at the fusion center. This special case of independent measurements in fact, turns out to be a lower bound for energy consumption of any data fusion scheme.

Lemma 1 (Lower Bound for $\mathrm{C}_{*}$ ): The minimum energy consumption $\mathrm{C}_{*}$ for a data fusion scheme that delivers the LLR in

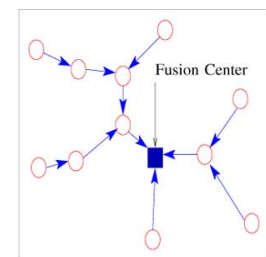

(a) MST is optimal for i.i.d. data.

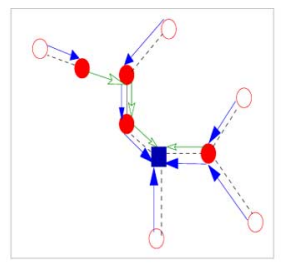

(b) DFMRF routing for detection of MRF.

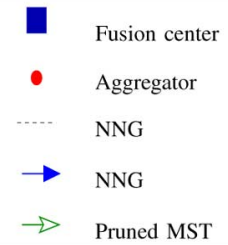

(c) Legend
Fig. 3. Routing schemes for detection under different correlation models.

(17) to the fusion center is at least the total energy consumption along the minimum spanning tree, i.e.

$$
\mathrm{C}_{*} \geq \mathrm{C}(\mathrm{MST}) .
$$

The lower bound is achieved iff. the sensor data are independent, conditioned on either hypotheses.

Hence, the presence of correlation increases routing costs since the log-likelihood ratio in (17) is more complicated. In [28], we show that incorporating correlation makes minimum energy routing a NP-hard problem and, hence, we have to focus on approximation algorithms. We employ a 2-approximation routing algorithm called the DFMRF, proposed in [3], and we restate its approximation guarantee here.

Lemma 2 (Approx. Ratio for DFMRF): DFMRF is a 2-approximation algorithm for any arbitrary node placement.

$$
\frac{\mathrm{C}(\mathrm{DFMRF})}{\mathrm{C}_{*}} \leq 2 .
$$

Hence, for any arbitrary node placement, the energy consumption of the DFMRF scheme is at most twice that of the optimal fusion scheme. Since we consider random point sets here, the approximation ratio can be improved by analyzing the asymptotic energy consumption and we undertake it in Section VII.

The DFMRF scheme proceeds in two stages. In the first stage, sensor measurements are transmitted along the edges of the NNG. This ensures that the edge potentials in (17) are computed at various nodes (called the aggregators). In the next stage, the computed values are summed up along the edges of the pruned minimum spanning tree (MST), where pruning refers to the removal of leaves. Details about the DFMRF scheme can be found in [3]. A schematic of the DFMRF scheme is shown in Fig. 3(b).

$$
\begin{aligned}
\operatorname{LLR}\left(\mathbf{Y}_{n}, \mathcal{G}_{d}\right)= & n \log \frac{\sigma_{1}}{\sigma_{0}} \\
+ & +\frac{1}{2}\left[\sum_{i \in \mathcal{V}}\left(\frac{1}{\sigma_{1}^{2}}-\frac{1}{\sigma_{0}^{2}}\right) Y_{i}^{2}+\sum_{(i, j) \in \mathcal{E}}\left\{\log \left[1-g^{2}\left(R_{i j}\right)\right]\right.\right. \\
& \left.\left.\quad+\frac{g^{2}\left(R_{i j}\right)}{1-g^{2}\left(R_{i j}\right)} \frac{Y_{i}^{2}+Y_{j}^{2}}{\sigma_{1}^{2}}-\frac{2 g\left(R_{i j}\right)}{1-g^{2}\left(R_{i j}\right)} \frac{Y_{i} Y_{j}}{\sigma_{1}^{2}}\right\}\right]
\end{aligned}
$$




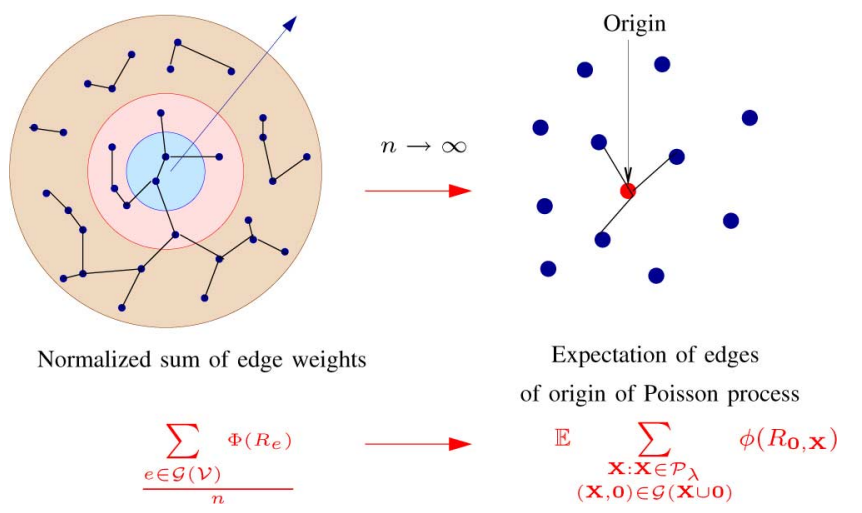

Fig. 4. Pictorial representation of LLN for graph functionals of uniform or Poisson point sets.

From (4), the total energy consumption of the DFMRF scheme can be expressed as

$$
\begin{aligned}
\mathrm{C}(\mathrm{DFMRF}) & =n+C_{t} \\
\times & \left(\sum_{e \in \mathrm{NNG}} R_{e}^{\nu}+\sum_{e \in \mathrm{MST}} R_{e}^{\nu}-\sum_{e \in \mathcal{L}_{f}(\mathrm{MST})} R_{e}^{\nu}\right)
\end{aligned}
$$

where $\mathcal{L}_{f}(\mathrm{MST})$ represents the leaves of the MST.

\section{ASYMPTOTIC ANALYSIS}

It is intractable to analytically evaluate energy consumption or detection performance for an arbitrary node set. Therefore, we assume that the nodes are placed randomly, according to a point process defined on expanding regions, formally defined below. The number of nodes $n$ goes to infinity, with fixed node density $\lambda$ [Fig. 1(a)].

Definition 2 (Poisson and Binomial Processes): Let $\left(\mathcal{B}_{n}\right)_{n \geq 1}$ denote a sequence of squares or circles of area $n / \lambda$, centered at the origin, for any $\lambda>0$. A binomial point process on $\mathcal{B}_{n}$, denoted by $\mathcal{U}_{n, \lambda}$, consists of $n$ points distributed i.i.d. uniformly on $\mathcal{B}_{n}$. A homogeneous Poisson process of intensity $\lambda$ on $\mathcal{B}_{n}$, denoted by $\mathcal{P}_{n, \lambda}$, satisfies the following properties:

1) for any set $\mathcal{A} \subset \mathcal{B}_{n}$ with area $A$, the number of points in $\mathcal{A}$ is Poisson distributed with mean $\lambda A$,

2) for any $n \in \mathbb{N}$ and $\mathcal{A} \subset \mathcal{B}_{n}$ with area $A>0$, conditioned on $n$ number of points in $\mathcal{A}$, the point process on $\mathcal{A}$ is a binomial process.

Such a random node placement allows us to employ the LLN for graph functionals, briefly described here.

\section{A. LLN for Graph Functionals}

The LLN for functionals on graphs enables us to evaluate the error exponent and applies to graphs which are random in the sense that the vertex set is a marked random point set. LLN on graphs is based on the so-called objective method. Steele [37] coined this term for a philosophy whereby, loosely speaking, one describes the limiting behavior of functionals on finite point sets of binomial process in terms of related functionals defined on infinite Poisson point sets. Penrose and Yukich [31], [34], [38] introduced a concept of stabilizing functionals and use the objective method to establish a strong law of large numbers for graph functionals [31, p. 287]. The result is illustrated in Fig. 4, where the limit of empirical average of the edge functionals of a stabilizing graph is the expectation over the sum of only the edge functionals of the origin, with the points drawn from an infinite Poisson process. Hence, the LLN states that the limit is a localized effect around the origin. The LLN is valid for stabilizing graphs, where the local behavior of the graph in a bounded region is unaffected by points beyond a finite (but random) distance from that region. The NNG is one such stabilizing graph with translation and scale-invariance [34, Lemma 6.1] and we focus on NNG dependency in this paper.

\section{B. Average Energy for DFMRF}

We now analyze the average energy consumption for the DFMRF scheme. The average energy for DFMRF under the binomial or Poisson process can be quantified using the LLN for graph functionals [31].

Theorem 2 (Optimal Average Energy): The asymptotic average energy consumption for the DFMRF scheme and for fusion along the minimum spanning tree (MST) are given by

$$
\begin{aligned}
\lim _{n \rightarrow \infty} \frac{\mathrm{C}(\operatorname{DFMRF}(\lambda))}{n} & =C_{t} \lambda^{-\nu / 2} c_{1}(\nu) \\
\lim _{n \rightarrow \infty} \frac{\mathrm{C}(\operatorname{MST}(\lambda))}{n} & =C_{t} \lambda^{-\nu / 2} c_{2}(\nu)
\end{aligned}
$$

where $c_{1}(\nu)$ and $c_{2}(\nu)$ are constants, independent of $\lambda$, and $\nu$ is the channel path-loss coefficient in (4). Hence, the optimal average energy consumption for data fusion $\overline{\mathrm{C}}_{*}$ satisfies the bounds

$$
\begin{aligned}
\lim _{n \rightarrow \infty} \frac{\mathrm{C}(\operatorname{MST}(\lambda))}{n} \leq \overline{\mathrm{C}}_{*}(\lambda) \leq \lim _{n \rightarrow \infty} \frac{\mathrm{C}(\operatorname{DFMRF}(\lambda))}{n} \\
C_{t} \lambda^{-\nu / 2} c_{2}(\nu) \leq \overline{\mathrm{C}}_{*}(\lambda) \leq C_{t} \lambda^{-\nu / 2} c_{1}(\nu) .
\end{aligned}
$$

This implies that the approximation ratio of the DFMRF under random node placement is improved to $c_{1}(\nu) / c_{2}(\nu)$.

Proof: The limit in (22) is proven in [38]. To evaluate the limit in (21), we use the expression in (20). We note that the power-weighted edges of the NNG, MST, and the leaves of MST are all stabilizing graph functionals [38]. Therefore, the LLN for graph functionals holds and we have (21). For approximation ratio, we have

$$
\lim _{n \rightarrow \infty} \frac{\mathrm{C}(\mathrm{DFMRF})}{\mathrm{C}_{*}} \leq \lim _{n \rightarrow \infty} \frac{\mathrm{C}(\mathrm{DFMRF})}{\mathrm{C}(\mathrm{MST})}=\frac{c_{1}(\nu)}{c_{2}(\nu)} .
$$

The constants $c_{1}(\nu)$ and $c_{2}(\nu)$ are the same for both the binomial and the Poisson process, and are evaluated through simulations. For a 500-node network, we obtained $c_{1}(2)=0.7134$, $c_{1}(3)=0.6462$, and $c_{1}(4)=0.6528$. Similarly, for the lower bound $c_{2}(\nu)$, which is based on the MST, analysis and simulation results are available in [39].

In Fig. 5, we plot simulation results comparing the average energy consumption of the DFMRF scheme $\left(c_{1}\right)$ and the lower bound $\left(c_{2}\right)$, based on the average energy consumption for fusion along the MST. We also plot the energy consumption for the classical approach using shortest paths to route all the raw data and we see that it does not have a constant-factor scaling. In 


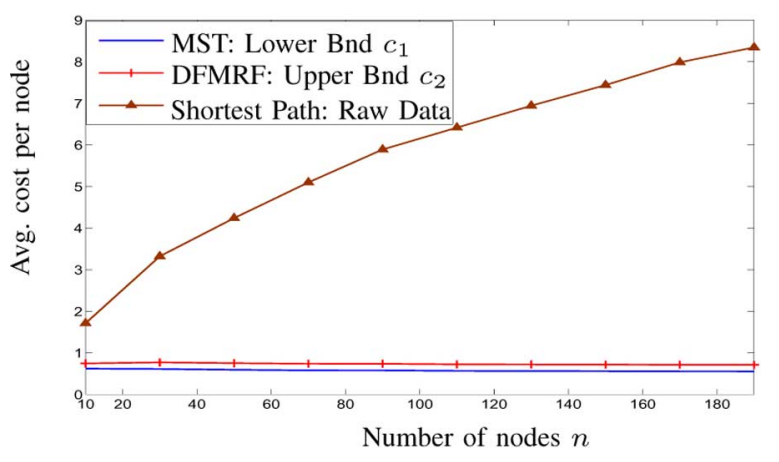

Fig. 5. Average per-node energy consumption for DFMRF scheme and shortest-path routing for nearest-neighbor dependency and uniform distribution. 500 simulation runs. Path-loss coefficient $\nu=2, C_{t}, \lambda=1$. See (4) and (23).

fact, as the number of nodes grows the average energy consumption for shortest-path routing grows almost linearly. Hence, collecting all the raw data from a large network with energy constrained nodes is not feasible and our fusion scheme DFMRF is applicable in such scenarios.

\section{Detection Error Exponent}

In this section, we characterize the detection error exponent for the hypothesis testing problem in (7). Under the NeymanPearson criterion, for a fixed Type-I error bound, the exponent of the Type-II error is independent of the type-I error bound [17], and is given by

$$
D:=\lim _{n \rightarrow \infty} \frac{1}{n} \log \frac{p\left[\mathbf{Y}_{n} ; \mathcal{H}_{0}\right]}{p\left[\mathbf{Y}_{n} \mid \mathcal{V} ; \mathcal{H}_{1}\right]}, \quad \text { under } \mathcal{H}_{0}
$$

In the following theorem, we restate the closed form for the error exponent, derived ${ }^{3}$ in [2], in terms of the variables and functions defined here.

$$
\begin{aligned}
f_{1}(x) & :=\log \left[1-x^{2}\right], f_{2}(x):=\frac{2 x^{2}}{\left[1-x^{2}\right]} \\
f(x, K) & :=f_{1}(x)+\frac{1}{K} f_{2}(x) \\
h_{i}(x ; g) & :=f_{i}(g(x))-\frac{\pi}{2 \omega} f_{i}\left(g\left(\sqrt{\frac{\pi}{\omega}} x\right)\right) \\
h(x, K ; g) & :=h_{1}(x ; g)+\frac{1}{K} h_{2}(x ; g)
\end{aligned}
$$

where $g(\cdot)$ is the correlation function. Let $Z$ denote the Rayleigh random variable with variance $(2 \pi)^{-1}$ and let $\omega$ be the area of the union of two unit- radii circles with centers unit distance apart, given by

$$
\omega=\frac{4 \pi}{3}+\frac{\sqrt{3}}{2} \approx 5.06
$$

Theorem 3 (Expression for d): For a GMRF on NNG with correlation function $g$, with the nodes drawn from the binomial

\footnotetext{
${ }^{3}$ The expression given in [2] is in a different form, but reduces to (30).
}

or the Poisson process with node density $\lambda$ and region area $n / \lambda$, the error exponent $D$ for Neyman-Pearson detection is

$$
D(\lambda, K ; g)=\frac{1}{2}\left[\mathbb{E}_{\lambda} h\left(Z \lambda^{-0.5}, K ; g\right)+\log K+\frac{1}{K}-1\right]
$$

where $\mathbb{E}_{\lambda}$ is the expectation over the random variable $Z$.

Proof: An intermediate expression for $D$ derived in [2] is given by

$$
\begin{aligned}
D(\lambda, K ; g)=\frac{1}{2}\left[\lim _{n \rightarrow \infty} \frac{1}{n} \sum_{e \in \mathrm{NNG}}\right. & \left.f\left(R_{e}\right), K ; g\right) \\
& \left.+\log K+\frac{1}{K}-1\right] .
\end{aligned}
$$

We then employ the special law of large numbers for graph functionals [31] to obtain (30). Details can be found in [2].

Note that in (30), the expectation term captures the correlation structure of the GMRF and the remaining terms represent the detection error exponent for two i.i.d. Gaussian processes with variance ratio $K$, i.e.

$$
D(\lambda, K ; g)=\frac{1}{2} \mathbb{E}_{\lambda} h\left(Z \lambda^{-0.5}, K ; g\right)+D_{\mathrm{IID}}(K)
$$

where $D_{\mathrm{IID}}(K)$ the error exponent when the measurements are conditionally i.i.d., and is given by

$$
D_{\text {IID }}(K)=\frac{1}{2}\left[\log K+\frac{1}{K}-1\right] .
$$

Hence, the effect of correlation on the error exponent is quantified in a compact form. It can be easily verified that the expectation term is zero, when $g(0)=M=0$ (no correlation). It is easy to see that $D_{\mathrm{IID}}(K)$ is independent of the node density $\lambda$. Hence, the issue of optimal node density for error exponent arises only in the presence of correlation. Moreover, intuitively, as we vary node density $\lambda$, the edge lengths in the resulting NNG scale by the factor $\lambda^{-0.5}$, on an average. Hence, we see that this factor $\lambda^{-0.5}$ appears in the expectation term in (32).

\section{OPtimal Node Density}

Recall that our objective is to find an optimal node density $\lambda_{*}$ maximizing the detection error exponent under a constraint on the average energy consumption of data fusion.

$$
\lambda_{*}:=\arg \max _{\lambda>0} D_{\lambda} \quad \text { subject to } \overline{\mathrm{C}}(\lambda) \leq \bar{E} .
$$

We have so far derived a closed-form expression for the error exponent $D_{\lambda}$ [short form for $D(\lambda, K ; g)$ in (30)] and bounds for optimal average energy consumption $\overline{\mathrm{C}}_{*}(\lambda)$ in (23). In this section, we exploit these expressions to derive the optimal node density.

Before proceeding to the actual derivations, it is useful to consider a special case, viz., when both the hypotheses have the same measurement variance $\left(K=\left(\sigma_{1} / \sigma_{0}\right)^{2}=1\right)$ and there is no energy constraint $(\bar{E} \rightarrow \infty)$. Since there is no correlation under $\mathcal{H}_{0}$, the two hypotheses can be distinguished only by the presence of correlation under $\mathcal{H}_{1}$. Correlation is maximized 
when all the nodes are clustered close to one another, since correlation decays with distance. Hence, the optimal density should be infinite. We prove that this is indeed true and also characterize the optimal density for general $K$ and energy constraint $\bar{E}$. To this end, we first characterize the set of feasible node densities which can support data fusion under the given energy constraint $\bar{E}$.

\section{A. Feasible Node Density Set}

In order to incorporate the energy constraint $\bar{E}$, we need to find a feasible set $\Lambda$ of node densities for minimum energy routing that satisfies the average energy constraint $\bar{E}$ and delivers the LLR to the fusion center,

$$
\Lambda(\bar{E}):=\left\{\lambda: \overline{\mathrm{C}}_{*}(\lambda) \leq \bar{E}\right\} .
$$

When the density goes to infinity, the optimal average energy consumption for routing goes to zero

$$
\lim _{\lambda \rightarrow \infty} \overline{\mathrm{C}}_{*}(\lambda)=0 .
$$

This is derived from the bounds on $\overline{\mathrm{C}}_{*}$ in (23). Hence, the energy constraint $\bar{E}$ is satisfied at infinite density or in other words, $\lambda \rightarrow \infty \in \Lambda$ for $\Lambda$ defined in (34). Hence, whenever $\Lambda$ is nonempty, it is of the form

$$
\Lambda(\bar{E})=\left[\lambda_{\bar{E}}, \infty\right]
$$

where $\lambda_{\bar{E}}$ is defined as the minimum node density under the energy constraint $\bar{E}$ at which it is feasible to perform data fusion. However, as discussed in Section VI, finding the minimum energy scheme is NP-hard. Hence, finding an expression for $\lambda_{\bar{E}}$ is analytically intractable. We instead provide bounds on $\lambda_{\bar{E}}$ using (23).

We first consider feasible node density for DFMRF scheme. From (21), imposing the energy constraint $\bar{E}$ translates to a constraint on $\lambda$ under the DFMRF

$$
\begin{aligned}
\overline{\mathrm{C}}(\operatorname{DFMRF}(\lambda)) & \leq \bar{E} \Longleftrightarrow \lambda \geq \lambda_{1}(\bar{E}) \\
& :=\left(\frac{\bar{E}}{C_{t} c_{1}(\nu)}\right)^{2 / \nu} .
\end{aligned}
$$

Similarly, from (22), imposing the constraint $\bar{E}$ for data fusion along the edges of the MST leads

$$
\overline{\mathrm{C}}(\operatorname{MST}(\lambda)) \leq \bar{E} \Longleftrightarrow \lambda \geq \lambda_{2}(\bar{E}):=\left(\frac{\bar{E}}{C_{t} c_{2}(\nu)}\right)^{2 / \nu} .
$$

From the bounds on optimal average energy in (23), we have bounds on the feasible set of node densities $\Lambda$ for the minimum energy scheme

$$
\left\{\lambda: \lambda \geq \lambda_{2}(\bar{E})\right\} \subset \Lambda(\bar{E}) \subset\left\{\lambda: \lambda \geq \lambda_{1}(\bar{E})\right\} .
$$

From the definition of $\lambda_{\bar{E}}$ in (35), we have

$$
\lambda_{2}(\bar{E}) \leq \lambda_{\bar{E}} \leq \lambda_{1}(\bar{E}) .
$$

Hence, although we are unable to evaluate $\lambda_{\bar{E}}$, we have bounds that are easily evaluated.

\section{B. Infinite Node Density}

We first analyze the error exponent $D$, when the node density $\lambda$ goes to infinity. This will provide insights for finding the optimal density. As $\lambda \rightarrow \infty$, we have

$$
D(\infty, K ; g)=D_{\mathrm{IID}}(K)+\frac{1}{2} h(0, K, M)
$$

where $h(0, K ; g)$ depends on $g$ only through $g(0)=M$, and

$$
h(0, K, M)=\left(1-\frac{\pi}{2 \omega}\right)\left(\log \left[1-M^{2}\right]+\frac{2 M^{2}}{K\left[1-M^{2}\right]}\right) .
$$

In the theorem below, we prove that the presence of correlation can either improve or degrade the error exponent, depending on the variance ratio $K$. We establish a threshold on $K$ that determines the transition.

Lemma 3 (Behavior at Infinite Density $(\lambda \rightarrow \infty)$ ): At $\lambda \rightarrow$ $\infty$, the correlation term $h(0, K, M)$ in (40) is positive, if the variance ratio $K$ is below a threshold value $K_{t}(M)$,

$$
h(0, K, M)\left\{\begin{array}{l}
\geq 0, \text { for } K<K_{t}(M) \\
<0, \text { for } K<K_{t}(M) .
\end{array}\right.
$$

For a fixed $g(0)=M<1$, the threshold $K_{t}(M)$ is

$$
K_{t}(M)=-\frac{1}{\log \left(1-M^{2}\right)} \frac{2 M^{2}}{1-M^{2}}
$$

and $2<K_{t}(M)<2 / 1-M^{2}$.

Proof: From (40) and $(1-\pi / 2 \omega)>0$.

Hence, we obtain a somewhat surprising result that at infinite node density, the effect of correlation on error exponent is different based on the variance ratio $K$ and is determined by a threshold $K_{t}$ on $K$. For values of $K$ below the threshold $K_{t}$, the presence of correlation improves the error exponent in (41a). On other hand, above the threshold $K_{t}$, the presence of correlation degrades the error exponent in (41b). Moreover, at infinite density since the internode distances go to zero, the correlation function is given by $g(0)=M$, and, hence, the threshold $K_{t}$ in (41) is only a function of $M$.

Although the results in Lemma 3 are valid only at infinite density, we can utilize them to compare with the other extreme scenario when the density $\lambda \rightarrow 0$. In this case, the error exponent $D_{\lambda} \rightarrow D_{\text {IID }}$, i.e., the conditionally i.i.d. case. From Lemma 3, we can conclude that below the threshold $K_{t}$, it is better to cluster the nodes close to one another $(\lambda \rightarrow \infty)$ rather than place them as far as possible $(\lambda \rightarrow 0)$. On the other hand, above the threshold, the opposite is true. Hence, the results for infinite node density in Lemma 3 provide guidelines on the effect of correlation on the error exponent. In Section VIII-C, we will generalize these results to prove that the optimal node density displays a threshold behavior.

\section{Threshold Behavior of Optimal Density}

In this section, we provide the results for optimal density. From (35), we can rewrite density optimization in (1) as

$$
\lambda_{*}=\arg \max _{\lambda \geq \lambda_{\bar{E}}} D_{\lambda}=\arg \max _{\lambda \geq \lambda_{\bar{E}}} \mathbb{E}_{\lambda} h\left(Z \lambda^{-0.5}, K ; g\right)
$$

where for the last equality, we use the fact that $D_{\mathrm{IID}}(K)$ is independent of $\lambda$ in (32). 
To analyze the behavior of the expectation term in (43), we first focus on the function $f$ in (26) given by

$$
f(x, K)=\log \left(1-x^{2}\right)+\frac{2}{K} \frac{x^{2}}{1-x^{2}}, \quad \forall x \in[0, M] .
$$

Since $g(0)=M$ and $g(\infty)=0$, we have $x \in[0, M]$. In the lemma below, we provide results on the behavior of $f$.

Lemma 4 (Behavior of $f$ ): The function $f(x, K)$ in (44) with $M<1$ satisfies

$$
\arg \max _{x \in[0, M]} f(x, K)= \begin{cases}M, & K<K_{t} \\ 0, & \text { o.w. }\end{cases}
$$

Proof: See Appendix A.

Hence, the function $f(x, K)$ attains its maximum only at one of the boundary points for $x \in[0, M]$. The particular boundary point is determined by a threshold $K_{t}$ on $K$, as seen in (45) and also $K_{t}$ depends only on $M$, the limiting correlation.

When the point sets are drawn from binomial or Poisson processes, and $R_{e}$ are the edge-lengths of NNG, consider the edge functional

$$
\frac{1}{n} \sum_{e \in \mathrm{NNG}} f\left(g\left(R_{e}\right), K ; g\right) .
$$

From (30) and (31), we have

$$
\lim _{n \rightarrow \infty} \frac{1}{n} \sum_{e \in \mathcal{E}_{d}} f\left(g\left(R_{e}\right), K ; g\right)=\mathbb{E}\left[h\left(Z \lambda^{-0.5}, K ; g\right)\right] .
$$

Hence, we can use the result on the maximum of function $f$ in Lemma 4 to find the corresponding optimal density maximizing the expectation term in (43). In the theorem below, we provide such a result on the optimal density and show that its behavior is determined only by the thresholds $K_{t}$ and $K_{t}^{\prime}$ on $K$.

Theorem 4 (Result on $\lambda_{*}(K, \bar{E})$ ): The optimal density in (43) that maximizes the error exponent, under feasible average energy constraint $\bar{E}$, is given by

$$
\lambda_{*}(K, \bar{E})=\infty, \quad \forall K<K_{t}(M)
$$

where the threshold $K_{t}$ is given by (42), and

$$
\lambda_{*}(K, \bar{E})=\lambda_{\bar{E}}<\infty, \quad \forall K>K_{t}^{\prime}(M)
$$

where $\lambda_{\bar{E}}$ is defined in (35), and satisfies bounds in (39), and

$$
K_{t}^{\prime}(M)=\frac{2}{1-M^{2}}>K_{t}(M)
$$

where $M=g(0)<1$ is the correlation function as the internode distance goes to zero. Also, when the energy constraint is infinite $\bar{E} \rightarrow \infty$, we have $\lambda_{\bar{E}}=0$, and the result in (48) is improved to

$$
\lambda_{*}(K, \infty)=0, \quad \forall K>K_{t}(M) .
$$

Proof: See Appendix B.

The above theorem states that when the variance ratio $K$ is below the threshold $K_{t}$, for any feasible energy constraint $\bar{E}$, optimality is attained at infinite density. On the other hand, above another threshold $K_{t}^{\prime}>K_{t}$, the minimum feasible node

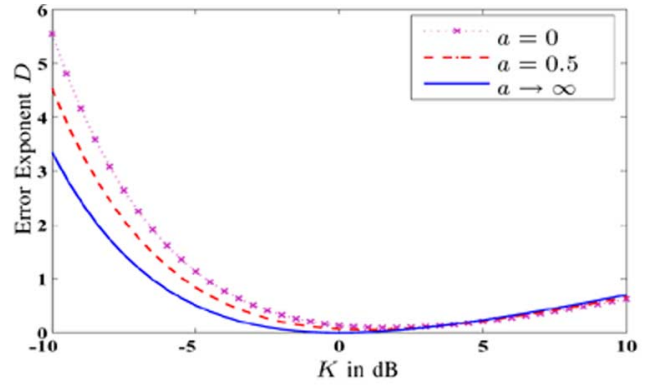

(a) Different values of correlation coefficient $a$, limiting correlation $M=0.5$.

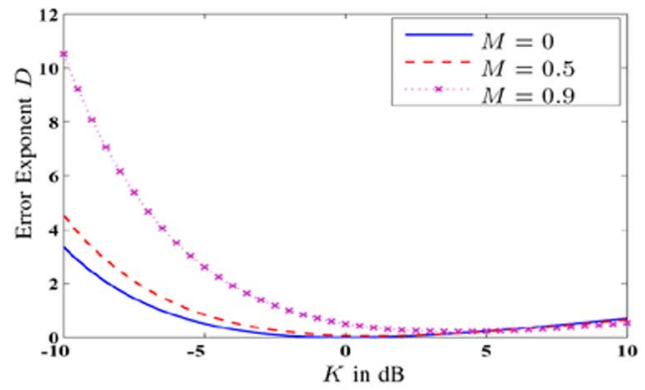

(b) Different values of limiting correlation $M$, correlation coefficient $a=0.5$.

Fig. 6. Error exponent $D$ versus ratio of variances $K$ for exponential correlation function, node density $\lambda=1$. See (30) and (51).

density $\lambda_{\bar{E}}$ which supports data fusion under constraint $\bar{E}$ attains the optimal value.

In the special case, when there is infinite energy $(\bar{E} \rightarrow \infty)$, we have $\lambda_{1}(\bar{E})=\lambda_{2}(\bar{E})=\lambda_{\bar{E}}=0$. In this case, we prove that optimal value is zero even in the gap region between the two thresholds $K_{t}$ and $K_{t}^{\prime}$. Numerical investigation suggests that under any finite energy constraint, the optimal density is $\lambda_{\bar{E}}$ even in the gap region between the two thresholds $K_{t}$ and $K_{t}^{\prime}$.

Also interestingly, the thresholds $K_{t}$ and $K_{t}^{\prime}$ depend on correlation $g(R)$ only through the limiting value $g(0)=M$. This is because of the behavior of the function $f$, elucidated in Lemma 4. We also note that as $M \rightarrow 1$, the thresholds $K_{t}, K_{t}^{\prime} \rightarrow \infty$. Hence, when we approach full correlation as the internode distance goes to zero, the optimal node density tends towards infinity for all values of $K$.

\section{Numerical Analysis}

In this section, we plot the error exponent and optimal node density for a specific correlation function namely the exponential-correlation function,

$$
g(R)=M \mathrm{e}^{-a R}, \quad a>0,0<M<1 .
$$

Using Theorem 3 , we numerically evaluate $D$ through Monte Carlo runs. In (30), the error exponent is an implicit function of the correlation coefficient $a$, through the correlation function $g$. We plot the effects of correlation coefficient $a$ and limiting correlation $M$ on $D$ in Fig. 6.

In Fig. 6(a), we plot the error exponent at $\lambda=1$ and $M=0.5$, for different values of correlation coefficient $a$. We notice that a more correlated GMRF or the one with smaller $a$, has a higher 


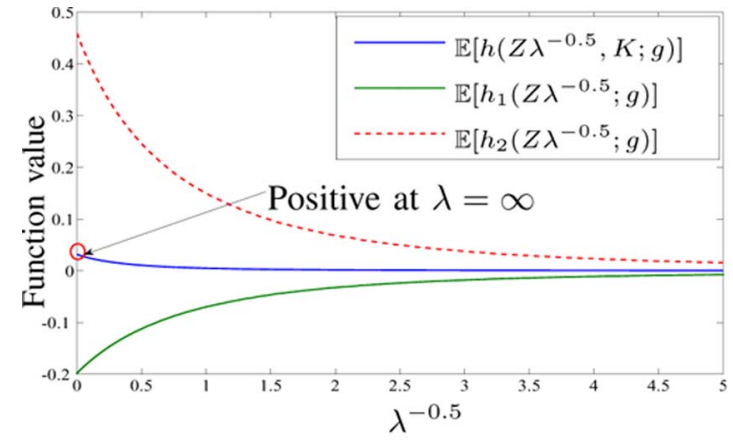

(a) Correlation provides positive contribution. $K=2<K_{t}$

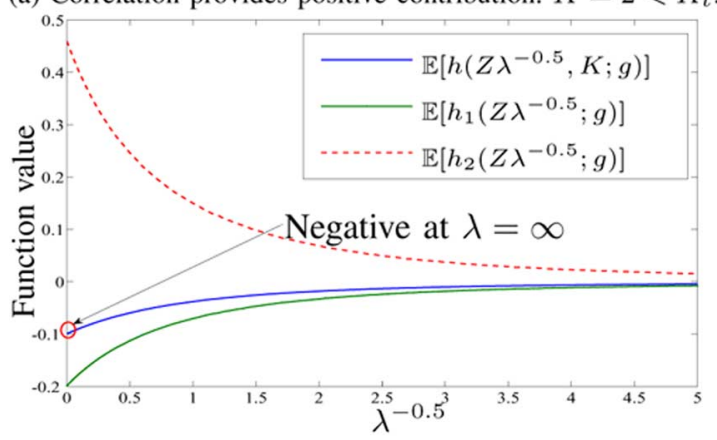

(b) Correlation provides negative contribution. $K=2 K_{t}$.

Fig. 7. Behavior of functions with exponential correlation $g(R)=$ $M \exp [-a R], M=0.5, a=1$. Functions $h_{1}$ and $h_{2}$ are independent of $K$. However, $K$ affects their scaling in $h$. See (28).

exponent at low value of $K$, whereas the situation is reversed at high $K$. Also, when the variance ratio $K$ is large enough, $D$ appears to increase linearly with $K$ (in $\mathrm{dB}$ ), and the correlation coefficient $a$ and the limiting correlation $M$ appear to have little effect, as expected from Theorem 3. In Fig. 6(b), we plot the exponent at constant correlation coefficient $a=0.5$ for different values of the limiting correlation $M$. Also note, $M=0$ reduces to the independent case. We notice a similar behavior as the correlation coefficient $a$ in Fig. 6(a). A higher value of $M$ results in a higher exponent at low $K$, but not at high $K$.

In Fig. 7, we fix the correlation coefficient $a=1$ in (51), and plot the expectations of functions $h, h_{1}$ and $h_{2}$ against $\lambda^{-0.5}$. In Fig. 7(a) and (b), the value of $K$ is below and above the threshold $K_{t}$. We observe that the behavior at $\lambda=\infty$ is different in the two plots. Note that the functions $h_{1}$ and $h_{2}$ are independent of $K$, but $K$ affects their scaling in $h$.

In Fig. 8(a) and (b), we numerically evaluate the optimal $\lambda_{*}\left(K, \lambda_{\bar{E}}\right)$ for different values of $M$ and variance ratio $K$. It is convenient to plot the results in terms of $\lambda^{-0.5}$, since the optimal $\lambda_{*}$ is infinite when $K$ is below the threshold $K_{t}$. We observe the threshold behavior at $K_{t}$, as predicted in Theorem 4: when $K<K_{t}$, we have $\lambda_{*}=\infty$ and for $K>K_{t}$, in Fig. 8(a) and (b), optimality is mostly attained at the other extreme point $\lambda_{\bar{E}}$. This is consistent with Theorem 4 .

\section{E. Sensitivity Analysis}

In Theorem 4, we proved the result on the optimal density $\lambda_{*}$. In this section, we analyze the extent to which the error exponent

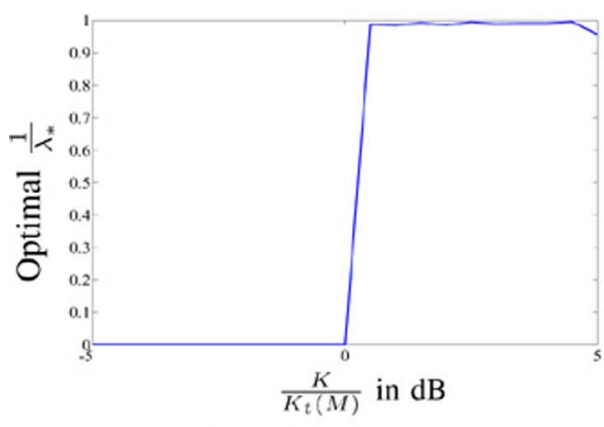

(a) $M=0.1$

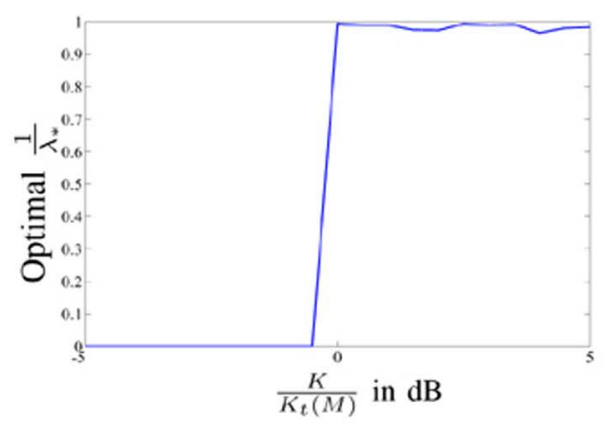

(b) $M=0.8$

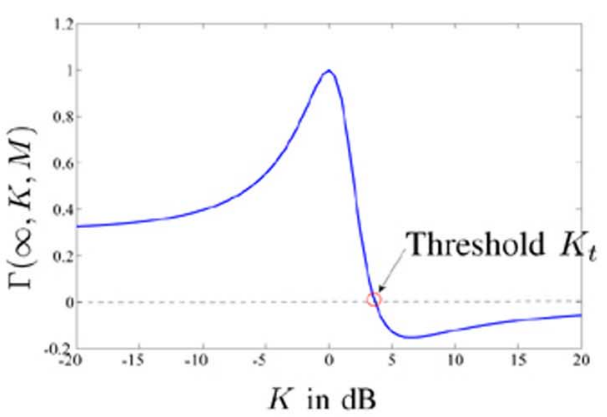

(c) Sensitivity analysis

Fig. 8. [a,b] The optimal density $\lambda_{*}$ is decided by threshold $K_{t}(M)$ on variance ratio $K \cdot \lambda_{\bar{E}}=1$. See Theorem 4 . [c] The contribution from correlation to error exponent versus variance ratio $K$ at $\lambda=\infty$ and $M=0.5$. See (52)

is dependent on $\lambda$. This enables us to gauge the usefulness of finding $\lambda_{*}$. To this end, we define

$$
\Gamma(\lambda, K ; g):=\frac{\mathbb{E}\left[Z \lambda^{-0.5}, K ; g\right]}{2 D(\lambda, K ; g)}
$$

which is the fraction of contribution coming from correlation to the error exponent in (32), and, hence, it is the part influenced by $\lambda$. Note, $0 \leq \Gamma(\lambda, K ; g) \leq 1$ and $\Gamma(0, K ; g)=0$.

Lemma 5 (Sensitivity of $d_{\lambda}$ to $\lambda$ ): At $K=1$, the fraction of contribution from correlation to the error exponent $\Gamma(\lambda, K ; g)$ is maximum

$$
\Gamma(\lambda, 1 ; g)=1, \quad \forall \lambda>0 .
$$

Also, in the large- $K$ regime

$$
\Gamma(\lambda, K ; g) \rightarrow 0, \quad \text { as } K \rightarrow \infty, \forall \lambda \in \Re^{+} .
$$


Hence, node density greatly influences detection performance at $K=1$. Intuitively, this is because at $K=1$, the two hypotheses can only be distinguished through the presence of correlation under $\mathcal{H}_{1}$. We also see that it decays to zero as $K \rightarrow \infty$. Hence, the error exponent is insensitive to changes in density at high $K$. In Fig. 8(c), we plot $\Gamma(\lambda, K ; g)$ as a function of $K$.

\section{ENERGY-DENSITY CONSTRAINT}

We now analyze the optimal node density under a different formulation. Instead of having fixed number of nodes placed in varying areas under a given constant node density, in this section, we fix areas of deployment $A$ according to a constant node density $\lambda$ and then, let $A \rightarrow \infty$. This means that we now have the flexibility of placing few powerful nodes or many cheaper nodes in a given area. On the other hand, earlier we had the option of choosing the area of deployment for a fixed number of nodes.

Under this formulation, the processing energy $C_{p}$ at each node needs to be incorporated. We impose an energy density constraint $\kappa$

$$
\kappa \geq \lim _{C, A \rightarrow \infty} \frac{C}{A}
$$

where $C$ is the total (routing + processing) energy consumption $C$ in area $A$. Hence, we impose a constraint on the energy consumption per unit area, instead of a constraint on the average energy consumption per node, as in (1).

The error exponent $D_{\lambda}^{p}$ with respect to total energy $C$ is

$$
D_{\lambda}^{p}:=\lim _{C \rightarrow \infty}-\frac{1}{C} \log P_{M}(C) .
$$

The optimal density $\lambda_{*}^{p}$ under the energy-density constraint is

$$
\lambda_{*}^{p}:=\max _{\lambda \in \Re^{+}} D_{\lambda}^{p}
$$

subject to the energy-density constraint $\kappa$ in (55).

Note that the error exponent $D_{\lambda}^{p}$ with respect to the total energy $C$ can be expressed as

$$
D_{\lambda}^{p}=\frac{D_{\lambda}}{\overline{\mathrm{C}}(\lambda)}
$$

where $D_{\lambda}$ is the error exponent with respect to number of nodes and $\bar{C}(\lambda)$ is the average energy per node, dealt in the previous sections. Similarly, the energy density constraint simplifies to

$$
\kappa \geq \lim _{C, A \rightarrow \infty} \frac{C}{A} \approx \lambda \frac{C}{\lfloor\lambda A\rfloor}=\lambda \overline{\mathrm{C}}(\lambda), \quad \lambda \in \Re^{+}
$$

since $\lfloor\lambda A\rfloor$ is the number of nodes and the approximation consists of ignoring the integer requirement for the number of nodes. We again define $\Lambda$ for this setup to be the set of the feasible node densities under minimum energy routing

$$
\Lambda:=\left\{\lambda: \lambda \overline{\mathrm{C}}_{*}(\lambda) \leq \kappa\right\} .
$$

In the theorem below, we show the finiteness of $\lambda_{p}^{*}$. We note that this result is on lines of the results in [25] for a one-dimensional GMRP.

Theorem 5 (Finite $\lambda_{*}^{p}$ ): The optimal density $\lambda_{*}^{p}$ in (57) exists whenever the set $\Lambda$ in (60) is nonempty and $\lambda_{*}^{p}$ is additionally finite if the per-node processing energy $C_{p}>0$.
Proof: Note that a compact set possesses a finite extremum point. To show that $\Lambda$ is a compact set, we first note that $\Lambda$ is a closed set. To show the bounded property, note that

$$
\lambda \overline{\mathrm{C}}(\lambda) \sim \lambda\left[C_{p}+c \lambda^{-\nu / 2}\right] \rightarrow \infty, \text { as } \lambda \rightarrow \infty .
$$

Hence, the constraint $\kappa$ in (59) is violated as $\lambda \rightarrow \infty$ and, hence, $\infty$ cannot be in $\Lambda$. Hence, $\Lambda$ is closed and bounded, i.e., compact. This implies that $\lambda_{*}^{p} \in \Lambda$ is finite.

In the above theorem, note that for the feasible set $\Lambda$ to be nonempty, the constraint $\kappa$ has to be sufficiently large. In other words, we need a large enough energy density $\kappa$ to support energy consumption involved in processing and routing of measurements.

We observe that there is no threshold effect when there is a energy-density constraint and the optimal node density $\lambda_{*}^{p}$ is always finite. This is in sharp contrast with the results in the previous section, where under the per-node energy constraint, $\lambda_{*}$ can be unbounded depending on the regime of $K$. This is because here, the energy-density constraint $\kappa$ limits the energy consumption in a given area thereby making infinite density infeasible. This implies that $\lambda_{*}^{p}$ cannot be unbounded. In the theorem below, we provide bounds for $\lambda_{*}^{p}$ when the variance ratio $K<2$, based on the monotonicity of the error exponent in this regime.

Theorem 6 (Optimal $\lambda_{*}^{p}$ ): Let $\lambda_{i}^{p}(\kappa)$ be the largest (positive real) root of the equation

$$
\lambda C_{p}+\lambda^{1-\nu / 2} C_{t} c_{i}(\nu)-\kappa=0, \quad \text { for } i=1,2 .
$$

where $c_{1}(\nu)$ and $c_{2}(\nu)$ are the energy-scaling constants in (23). If the per-node processing energy $C_{p}>0$, the variance ratio $K<2$ and the constraint $\kappa$ is such that both $\lambda_{1}^{p}(\kappa)$ and $\lambda_{2}^{p}(\kappa)$ exist then the optimal $\lambda_{*}^{p}$ satisfies

$$
\lambda_{2}^{p}(\kappa) \leq \lambda_{*}^{p} \leq \lambda_{1}^{p}(\kappa) .
$$

Proof: On the lines of the arguments in the previous section, it can be shown that $D_{\lambda}$ is increasing in $\lambda$ for $K<2$. From (23), $\overline{\mathrm{C}}_{*}(\lambda)$ is decreasing in $\lambda$. Hence, the exponent $D_{\lambda}^{p}$ in (56) is increasing in $\lambda$. From the energy-density constraint in (59) and Theorem 5, the feasible set $\Lambda$ is bounded. From the bounds on $\overline{\mathrm{C}}_{*}(\lambda)$ in (23), if the largest real roots of (61), for $i=1,2$, exist, then the maximum value in $\Lambda$ is bounded by these roots.

Hence, in Theorem 6, we obtain bounds on the optimal density $\lambda_{*}^{p}$ under energy-density formulation. We prove this by first showing that the error exponent $D_{\lambda}^{p}$ is increasing with $\lambda$ when the variance ratio $K<2$, as seen in Fig. 9. This implies that $\lambda_{*}^{p}$ is the largest feasible density under constraint $\kappa$ that supports the energy consumption for data fusion. In the end, we provide the bounds in (62) on this largest feasible density through the bounds for average energy consumption $\bar{C}_{\lambda}$ in (23). Although we analytically prove the bounds in (62) only when the variance ratio $K<2$, the behavior of the error exponent $D_{\lambda}^{p}$ in Fig. 9 suggests that the bound in (62) may be valid for all values of $K$.

\section{Conclusion}

The tradeoff between the energy consumption in data fusion and the resulting detection performance at the fusion center is 


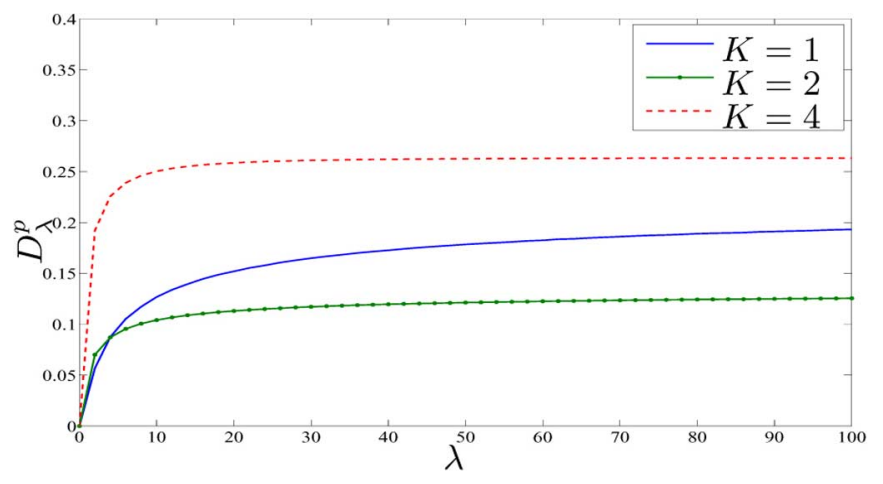

Fig. 9. The error exponent $D_{\lambda}^{p}$ with respect to the total energy. See (56). $\nu=2$, $C_{p}=1, C_{t}=1 / c_{1}(2), M=0.6$.

an important problem in the context of sensor networks. In this paper, we incorporated correlation between the measurements through the Gauss-Markov random field model. We characterized the density of node deployment that maximizes the detection error exponent subject to a constraint on the average energy consumption. The measurement variance is crucial in determining whether the optimal node density is limited by the fusion energy constraint and displays a threshold behavior. We derived the threshold analytically and verified it with simulations.

The results on the optimal density are possible due to exploitation of the Markovian structure, in deriving the error exponent and the fusion schemes. While acknowledging the limitations of its validity, we have made an attempt to characterize detection-energy tradeoffs for correlated measurements in two (and higher) dimensional spaces, which to the best of our knowledge, has not been dealt before.

Alternative formulations, not dealt in this paper, include selection of nodes with "useful" data [30], and incorporating node and link failures. We have also not considered the issue of quantization of measurements, a difficult problem even for conditionally i.i.d. measurements and the recent works in [40], [41] have considered it for tree and tandem network topologies. Another issue not addressed is that of the latency involved for the decision to be made at the fusion center. Extension of this work to incorporate more general dependencies among sensor measurements is currently under investigation.

\section{APPENDIX}

Proof of Lemma 4: Since $g(0)=M$ and $g(\infty)=0$, we have $x \in[0, M]$.

$$
\frac{\partial f}{\partial x}=\frac{2 x}{1-x^{2}}\left(-1+\frac{2}{K\left(1-x^{2}\right)}\right) .
$$

Therefore, $f$ has only one critical point in $(0, M]$. For $K<2$, $\partial f / \partial x>0$ and for $K>K_{t}^{\prime}, \partial f / \partial x<0, \forall x \in[0, M]$. There are no critical points. For $2<K<K_{t}^{\prime}$, the critical point is a minimum. Hence, maximum is attained at one of the boundary points $\{0, M\}$. For $K<K_{t}$, it is at $x=M=g(0)$ and, hence

$$
f\left(g\left(R_{e}\right), K\right) \leq f(g(0), K ; g), \quad \forall R_{e} \geq 0 .
$$

Similarly, for $K>K_{t}$, we have

$$
f\left(R_{e}, K ; g\right) \leq f(\infty, K ; g)=0, \quad \forall R_{e} \geq 0 .
$$

Proof of Theorem 4: From Lemma 4, when $K<K_{t}$, $g(0)=M$ attains the maximum of $f\left(g\left(R_{e}\right), K\right)$. Hence, we have for $R_{e} \geq 0$

$$
\frac{1}{n} \sum_{e \in \mathcal{E}_{d}} f\left(g\left(R_{e}\right), K ; g\right) \leq \frac{1}{n} \sum_{e \in \mathcal{E}_{d}} f(g(0), K ; g), \quad \forall K<K_{t} .
$$

Letting $n \rightarrow \infty$ on both sides, from (46)

$$
\mathbb{E}\left[h\left(Z \lambda^{-0.5}, K ; g\right)\right] \leq h(0, K, M), \quad \forall K<K_{t} .
$$

Hence, the optimal density in this regime is given by

$$
\lambda_{*}(K, \bar{E})=\arg \max _{\lambda \geq \lambda_{\bar{E}}} \mathbb{E}\left[h\left(Z \lambda^{-0.5}, K ; g\right)\right]=\infty \quad \forall K<K_{t} .
$$

From Lemma 4, when $K>K_{t}, g(\infty)=0$ attains the maximum of $f\left(g\left(R_{e}\right), K\right)$. For the case of infinite energy $\bar{E} \rightarrow \infty$, $\lambda_{\bar{E}}=\lambda_{2}(\bar{E})=\lambda_{1}(\bar{E})=0$. Hence

$$
\begin{aligned}
\lambda_{*}(K, \infty) & =\arg \max _{\lambda \geq \lambda_{\bar{E}}=0} \mathbb{E}\left[h\left(Z \lambda^{-0.5}, K ; g\right)\right] \\
& =0 \quad \forall K>K_{t} .
\end{aligned}
$$

For finite constraint $\bar{E}$ and $K>K_{t}^{\prime}, f\left(g\left(R_{e}\right), K\right)$ is increasing in $R_{e}$. We have $R_{e}=\lambda^{-0.5} R_{e}^{\prime}$, where $R_{e}^{\prime}$ is the edge-length in unit area and is independent of $\lambda$. Hence, $f\left(g\left(R_{e}\right), K\right)$ is nonincreasing in $\lambda$ and the limit $\mathbb{E}\left[h\left(Z \lambda^{-0.5}, K ; g\right)\right]$ is also nonincreasing in $\lambda$. Hence

$$
\begin{aligned}
& \lambda_{*}(K, \bar{E})= \\
& \quad \arg \max _{\lambda \geq \lambda_{\bar{E}}} \mathbb{E}\left[h\left(Z \lambda^{-0.5}, K ; g\right)\right]=\lambda_{\bar{E}} \quad \forall K>K_{t}^{\prime} .
\end{aligned}
$$

\section{ACKNOWLEDGMENT}

The authors would like to thank Prof. J. E. Yukich for extensive ongoing discussion regarding the LLN of graph functionals and Prof. Veeravali for pointing out the case of energy-density constraint. The authors also thank the anonymous reviewers and associate editor Prof. D. Mandic for comments that substantially improved this paper.

\section{REFERENCES}

[1] A. Anandkumar, L. Tong, and A. Swami, "Detection of Gauss-Markov random fields under routing energy constraint," in Proc. 45th Allerton Conf. Commun., Control Comput., Monticello, NY, Sep. 2007, pp. 1234-1238.

[2] A. Anandkumar, L. Tong, and A. Swami, "Detection of Gauss-Markov random fields with nearest-neighbor dependency," IEEE Trans. Inf. Theory, Dec. 2006, available on Arxiv, submitted for publication.

[3] A. Anandkumar, L. Tong, and A. Swami, "Energy efficient routing for statistical inference of Markov random fields," in Proc. CISS '07, Baltimore, MD, Mar. 2007, pp. 643-648.

[4] A. Deshpande, C. Guestrin, S. Madden, J. Hellerstein, and W. Hong, "Model-driven data acquisition in sensor networks," in Proc. VLDB, 2004.

[5] R. Cristescu and M. Vetterli, "On the optimal density for real-time data gathering of spatio-temporal processes in sensor networks," in Proc. IPSN, 2005, pp. 159-164.

[6] D. Marco, E. Duarte-Melo, M. Liu, and D. Neuhoff, "On the many-to-one transport capacity of a dense wireless sensor network and the compressibility of its data," in Proc. IPSN, 2003, pp. 1-16. 
[7] S. Yoon and C. Shahabi, "The clustered aggregation technique leveraging spatial and temporal correlations in wireless sensor networks," ACM Trans. Sens. Netw., vol. 3, no. 1, 2007.

[8] J. Faruque and A. Helmy, "Rugged: Routing on finGerprint gradients in sEnsor networks," in Proc. IEEE/ACS Int. Conf. Pervasive Services (ICPS), 2004, pp. 179-188.

[9] S. Pattem, B. Krishnamachari, and R. Govindan, "The impact of spatial correlation on routing with compression in wireless sensor networks," in Proc. 3rd Int. Symp. Inf. Process. Sens. Netw., 2004, pp. 28-35.

[10] J. Besag, "Spatial interaction and the statistical analysis of lattice systems," J. Royal Stat. Soc., vol. 36, no. B, pp. 192-225, 1974.

[11] J. Besag, "Statistical analysis of nonlattice data," The Statistician, vol. 24, no. 3, pp. 179-195, 1975.

[12] M. Cetin, L. Chen, J. Fisher, A. Ihler, O. Kreidl, R. Moses, M. Wainwright, J. Williams, and A. Willsky, "Graphical models and fusion in sensor networks," in Wireless Sensor Networks: Signal Processing And Communication Perspectives. New York: Wiley, 2007, ch. 9, pp. 215-250.

[13] S. Li, Markov Random Field Modeling in Computer Vision. London, U.K.: Springer-Verlag, 1995.

[14] N. Cressie, Statistics for Spatial Data. New York: Wiley, 1993.

[15] T. Kailath and H. Poor, "Detection of stochastic processes," IEEE Trans. Inf. Theory, vol. 44, no. 6, pp. 2230-2259, Oct. 1998.

[16] M. D. Donsker and S. R. S. Varadhan, "Large deviations for stationary Gaussian process," Commun. Math. Phys., vol. 97, pp. 187-210, 1985.

[17] P.-N. Chen, "General formulas for the Neyman-Pearson type-II error exponent subject to fixed and exponential type-I error bounds," IEEE Trans. Inf. Theory, vol. 42, no. 1, pp. 316-323, Jan. 1996.

[18] A. Giridhar and P. Kumar, "Toward a theory of in-network computation in wireless sensor networks," IEEE Commun. Mag., vol. 44, no. 4, pp. 98-107, 2006.

[19] R. Rajagopalan and P. Varshney, "Data aggregation techniques in sensor networks: A survey," IEEE Commun. Surveys Tutorials, vol. 8, no. 4, pp. 48-63, 2006.

[20] K. Akkaya and M. Younis, "A survey of routing protocols in wireless sensor networks," Elsevier Ad hoc Netw., vol. 3, pp. 325-349, 2005.

[21] Y. Yang and R. Blum, "Energy-efficient routing for signal detection under the Neyman-Pearson criterion in wireless sensor networks," in Proc. IPSN, 2007, pp. 303-312.

[22] S. Appadwedula, V. Veeravalli, and D. Jones, "Energy-efficient detection in sensor networks," IEEE J. Select. Area Comm., vol. 23, no. 4, pp. 693-702, 2005.

[23] L. Yu, L. Yuan, G. Qu, and A. Ephremides, "Energy-driven detection scheme with guaranteed accuracy," in Proc. IPSN, 2006, pp. 284-291.

[24] Y. Sung, S. Misra, L. Tong, and A. Emphremides, "Cooperative routing for signal detection in large sensor networks," IEEE J. Select. Area Comm., vol. 25, no. 2, pp. 471-483, 2007.

[25] J. Chamberland and V. Veeravalli, "How dense should a sensor network be for detection with correlated observations?", IEEE Trans. Inf. Theory, vol. 52, no. 11, pp. 5099-5106, 2006.

[26] N. Katenka, E. Levina, and G. Michailidis, "Local vote decision fusion for target detection in wireless sensor networks," in Proc. Joint Research Conf. on Statistics in Quality Industry and Tech., Knoxville, USA, Jun. 2006.

[27] O. Kreidl and A. Willsky, "Inference with minimal communication: a Decision-theoretic variational approach," in Proc. Advances in Neural Information Processing Systems, 2006.

[28] A. Anandkumar, L. Tong, A. Swami, and A. Ephremides, "Minimum cost data aggregation with localized processing for statistical inference," in Proc. IEEE INFOCOM, Phoenix, USA, Apr. 2008, pp. 780-788.

[29] A. Anandkumar, A. Ephremides, and L. S. TongA, "Minimum cost routing with local processing for distributed statistical inference," in Handbook on Array Processing and Sensor Networks, S. Haykin and R. Liu, Eds. New York: IEEE-Wiley, 2008, ch. 25.

[30] A. Anandkumar, L. Tong, A. Swami, and A. Ephremides, "Cost-performance tradeoff in multi-hop aggregation for statistical inference," in Proc. IEEE Int. Symp. Inf. Theory, Toronto, Canada, Jul. 2008.

[31] M. Penrose and J. Yukich, "Limit theory for random sequential packing and deposition," Ann. Appl. Probab., vol. 12, no. 1, pp. 272-301, 2002.

[32] H. Rue and L. Held, Gaussian Markov Random Fields: Theory and Applications. London: Chapman and Hall, 2005.

[33] A. Ephremides, "Energy concerns in wireless networks," IEEE Wireless Commun., no. 4, pp. 48-59, Aug. 2002.

[34] M. Penrose and J. Yukich, "Central limit theorems for some graphs in computational geometry," Ann. Appl. Probab., vol. 11, no. 4, pp. 1005-1041, 2001.
[35] J. Moura and N. Balram, "Recursive structure of noncausal GaussMarkov random fields," IEEE Trans. Inf. Theory, vol. IT-38, no. 2, pp. 334-354, Mar. 1992.

[36] J. Møller and R. Waagepetersen, Statistical Inference and Simulation for Spatial Point Processes. London, U.K.: Chapman \& Hall/CRC, 2004.

[37] J. Steele, Probability Theory and Combinatorial Optimization. Philadelphia, PA: Society for Industrial and Applied Mathematics, 1997.

[38] M. Penrose and J. Yukich, "Weak laws of large numbers in geometric probability," Ann. Appl. Probab., vol. 13, no. 1, pp. 277-303, 2003.

[39] M. Cortina-Borja and T. Robinson, "Estimating the asymptotic constants of the total length of Euclidean minimal spanning trees with power-weighted edges," Stat. Probab. Lett., vol. 47, no. 2, pp. 125-128, 2000.

[40] W. P. Tay, J. N. Tsitsiklis, and M. Z. Win, "Data fusion trees for detection: Does architecture matter?," IEEE Trans. Inf. Theory, pp. 4155-4168, Sep. 2008.

[41] W. P. Tay, J. N. Tsitsiklis, and M. Z. Win, "On the sub-exponential decay of detection error probabilities in long tandems," IEEE Trans. Inf. Theory, 2007, submitted for publication.

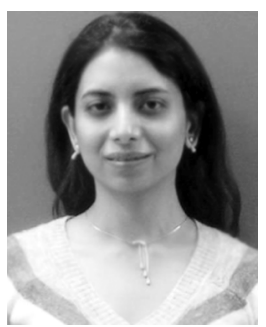

Animashree Anandkumar ( $\left.\mathrm{S}^{\prime} \mathrm{O} 2\right)$ received the B.Tech degree in Electrical Engineering from Indian Institute of Technology Madras, Chennai, India in 2004. She is currently pursuing her Ph.D. degree in Electrical Engineering at Cornell University, Ithaca, NY. She has been a member of the Adaptive Communications and Signal Processing Group (ACSP) at Cornell University since August, 2004.

Anima received the Fran Allen IBM Ph.D. fellowship for the year 2008-09, presented annually to one female $\mathrm{Ph} . \mathrm{D}$. student in conjunction with the IBM $\mathrm{Ph} . \mathrm{D}$. Fellowship Award, in recognition of her technical accomplishments, as well as her commitment to mentoring and community building. She was named a finalist for the Google Anita-Borg Scholarship 2007-08. She received the Student Paper Award at the 2006 International Conference on Acoustic, Speech and Signal Processing (ICASSP) held at Toulouse, France.

Anima Anandkumar's research interests are in the area of statistical-signal processing, information theory and networking. Specifically, she has been working on detection and estimation, asymptotic analysis and in-network function computation, in the context of wireless-sensor networks. She has served as a reviewer for IEEE TRANSACTIONS ON SigNAL PROCESSING, IEEE TRANSACTIONS ON INFORMATION THEORY, and various IEEE conferences.

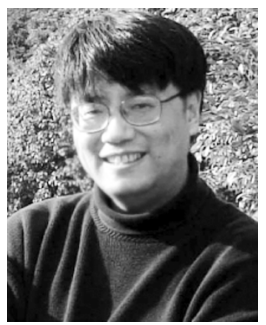

Lang Tong (S'87-M'91-SM'01-F'05) is the Irwin and Joan Jacobs Professor in Engineering at Cornell University Ithaca, New York. Prior to joining Cornell University, he was on faculty at the West Virginia University and the University of Connecticut. He was also the 2001 Cor Wit Visiting Professor at the Delft University of Technology. Lang Tong received the B.E. degree from Tsinghua University, Beijing, China, in 1985, and M.S. and Ph.D. degrees in electrical engineering in 1987 and 1991, respectively, from the University of Notre Dame, Notre Dame, Indiana. He was a Postdoctoral Research Affiliate at the Information Systems Laboratory, Stanford University in 1991.

Lang Tong is a Fellow of IEEE. He received the 1993 Outstanding Young Author Award from the IEEE Circuits and Systems Society, the 2004 best paper award (with Min Dong) from IEEE Signal Processing Society, and the 2004 Leonard G. Abraham Prize Paper Award from the IEEE Communications Society (with Parvathinathan Venkitasubramaniam and Srihari Adireddy). He is also a coauthor of five student paper awards. He received Young Investigator Award from the Office of Naval Research.

Lang Tong's research is in the general area of statistical signal processing, wireless communications and networking, and information theory. He has served as an Associate Editor for the IEEE TRAnsactions ON Signal PROCESSING, the IEEE TRANSACTIONS ON INFORMATION THEORY, and IEEE Signal Processing LetTers. 


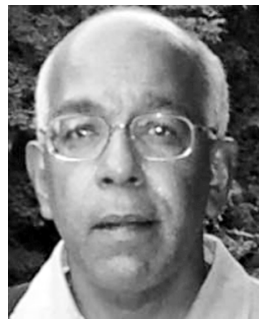

Ananthram Swami (S'79-M'81-SM'96-F'07) received the B.Tech. degree from IIT-Bombay; the M.S. degree from Rice University, Houston, TX, and the Ph.D. degree from the University of Southern California (USC), Los Angeles, all in electrical engineering.

He has held positions with Unocal Corporation, USC, CS-3, and Malgudi Systems. He was a Statistical Consultant to the California Lottery, developed a Matlab-based toolbox for non-Gaussian signal processing, and has held visiting faculty positions at INP, Toulouse, France. He is currently with the U.S. Army Research Laboratory where his work is in the broad area of signal processing, wireless communications, sensor and mobile ad hoc networks. He is a coeditor, with Q. Zhao,
Y.-W. Hong, and L. Tong, of Wireless Sensor Networks: Signal Processing \& Communications Perspectives (New York: Wiley, 2007).

Dr. Swami has served as an Associate Editor of the IEEE TRANSACTIONS on Wireless Communications, IEEE Signal PRocessing LetTers, IEEE TRANSACTIONS ON CIRCUITS AND SySTEMS-II, IEEE Signal Processing Magazine, and the IEEE TRANSACTIONS ON SIGNAL PROCESSING. He was a co-Guest Editor of a 2004 Special Issue (SI) of the IEEE Signal Processing Magazine (SPM) on Signal Processing for Networking, a 2006 SPM SI on Distributed Signal Processing in Sensor Networks, a 2006 EURASIP JASP SI on Reliable Communications over Rapidly Time-Varying Channels, a 2007 EURASIP $J W C N$ SI on Wireless Mobile ad hoc Networks, and is the Lead Editor for a 2008 IEEE JouRnAl OF SELECTED TOPICS IN Signal PROCESSING SI on Signal Processing and Networking for Dynamic Spectrum Access. 